\title{
Photon spin-to-orbital angular momentum conversion via an electrically tunable $q$-plate
}

Bruno Piccirillo, ${ }^{1,2}$, a) Vincenzo D'Ambrosio, ${ }^{1}$ Sergei Slussarenko, ${ }^{1}$ Lorenzo Marrucci, ${ }^{1,3}$ and Enrico Santamato ${ }^{1,2}$

1) Dipartimento di Scienze Fisiche, Università di Napoli "Federico II",

Complesso Universitario di Monte S. Angelo, 80126 Napoli, Italy

${ }^{2)}$ CNISM-Consorzio Nazionale Interuniversitario per le Scienze Fisiche della Materia,

Napoli

${ }^{3)}$ CNR-SPIN Complesso Universitario di Monte S. Angelo, 80126 Napoli, Italy

(Dated: 22 October 2010)

Exploiting electro-optic effects in liquid crystals, we achieved real-time control of the retardation of liquidcrystal-based $q$-plates through an externally applied voltage. The newly conceived electro-optic $q$-plates can be operated as electrically driven converters of photon spin into orbital angular momentum, enabling a variation of the orbital angular momentum probabilities of the output photons over a time scale of milliseconds.

For years the orbital angular momentum (OAM) of photons has been consigned to a back seat in the study of both classical and quantum optics. At the very beginning, in combination with the spin angular momentum (SAM), it was adopted as an essentially auxiliary concept describing the properties of wave functions under rotations ${ }^{1,2}$. This was partly due to the fact that, in general, the spin and orbital contributions cannot be considered separately ${ }^{2}$, except within the paraxial approximation. However, even in the small-angle limit, the OAM, due to the dearth of tools suitable for its manipulation, has been used less than SAM, the latter being much handier than the former thanks to the availability of a harvest of polarization optical components. No doubt, the interest for both fundamental and applicative aspects of OAM has kept up with the development of methods and devices for its manipulation. At present, the importance of OAM for quantum optics is mainly due to the fact that it is defined on an infinite-dimensional Hilbert space and, by its own nature, could be suitable for implementing single-photon qudits. The use of high dimensional qudits instead of qubits is desirable since it may, for example, lead to simplifying quantum computations $^{5,6}$ and improve quantum cryptography ${ }^{7}$. The most commonly used methods for generating and manipulating the OAM are based on computer generated holograms (CGHs). However, in order to manage the OAM of light as much handily as possible, it has been recently introduced a liquid-crystal-based birefringent plate with retardation $\delta$ and optical axis unevenly oriented according to a distribution with topological charge $q$, after which the name $q$-plate $(\mathrm{QP})^{8}$. A QP modifies the angular momentum state of an incident photon by giving to each of its circularly polarized components a finite probability, depending on the retardation $\delta$, of finding the photon in a polarization state with opposite helicity and OAM quantum number $l$ increased or decreased by the amount $\Delta l=2 q$, whether the initial helicity is positive or negative respectively. In $q$-plates with $q=1$, the optical axis

\footnotetext{
a) bruno.piccirillo@na.infn.it
}

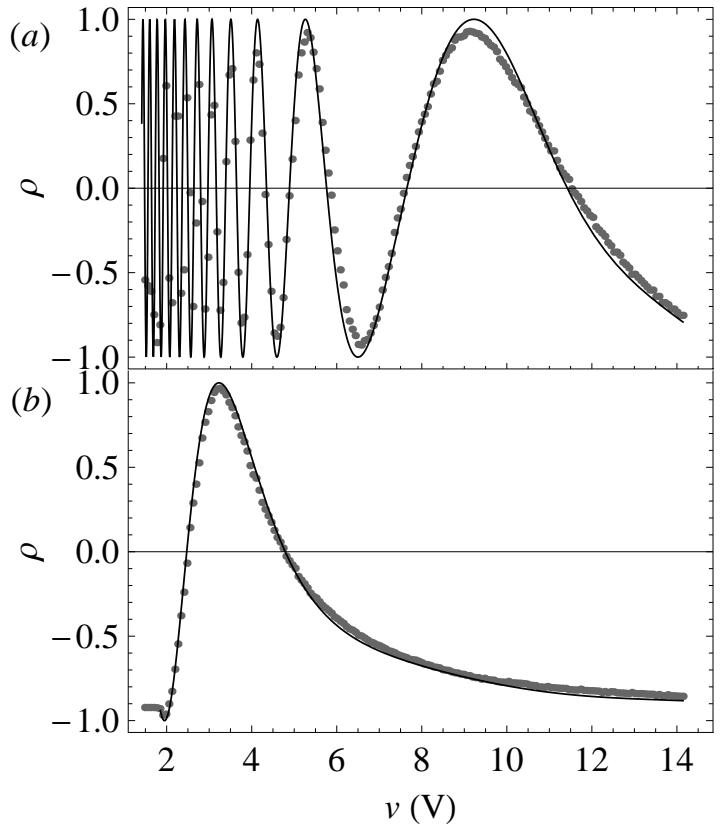

FIG. 1. Measured contrast ratio $\rho(v)=\cos \delta(v)$, reported as function of $v$ for $\operatorname{EOQP}_{1}(a)$ and $\operatorname{EOQP}_{2}(b)$. The continuous lines represent the theoretical behaviors.

distribution is cylindrically symmetric around the central defect and the total angular momentum of the incident photons is therefore conserved (SAM-to-OAM conversion or STOC). The adoption of such device in quantum optics has recently enabled the observation of two-photon HongOu-Mandel coalescence interference of photons carrying non-zero OAM and the demonstration of the $1 \rightarrow 2$ universal optimal quantum cloning of OAM-encoded qubits and qudits ${ }^{9-11}$. Besides the topological charge $q$, a keyfeature of a $\mathrm{QP}$ is the birefringent retardation, since it enables to regulate the probability of switching between $l$ and $l \pm 2 q$, i.e. the STOC efficiency, according to the wavelength of the input photons. High efficiencies for generation, manipulation and detection of OAM states are often desirable, especially when only few photons are available. 
In this paper, to achieve a full control of the STOC efficiency, we present an electro-optical $q$-plate (EOQP) whose retardation may be changed through an externally applied voltage. Hitherto, STOC efficiencies exceeding $95 \%$ (significantly higher than the efficiencies of the CGHs) were obtained tuning the $q$-plate retardation $\delta$ by controlling the material temperature ${ }^{12}$. A thermally-tuned QP has been successfully used with a Dove prism inserted into a Sagnac polarizing interferometer in order to generate arbitrary linear combinations of OAM eigenstates with $l= \pm 2$ by manipulating the polarization state of an input linearly polarized $\mathrm{TEM}_{00}$ laser beam ${ }^{13}$. The thermal tunability of $\delta$ arises from the temperature dependence of the liquid crystal order parameter, and ultimately of the intrinsic birefringence of such material. The thermal control of a QP assures an easy-to-made stable retardation at the cost of a very slow time response. This is a limitation whenever the experiment requires a real-time variation of STOC efficiency, for instance, for qudit manipulation or when more wavelengths are involved. Electric-field-based regulation of $\delta$ enables one to overcome the time-response limitations imposed by the thermal method and make QPs suitable for more demanding tasks. The working principle of EOQP is based on the well-known property of external static electric or magnetic fields to change the orientation of the liquid-crystal molecular director $\boldsymbol{n}$, representing the local average orientation of liquid crystal molecules ${ }^{14}$. We fabricated and tested two EOQPs with $q=1$, so that the OAM impressed to converted photons is $l= \pm 2$. These devices have different thickness and have been manufactured by different methods. The first was a nominal $20 \mu \mathrm{m}$ thick film of E7 liquid crystal from Merck Ltd, sandwiched between two ITOcoated glass substrates, beforehand coated with a polyimide for planar alignment and circularly rubbed, as described elsewhere ${ }^{12}$. The second EOQP was a nominal $6 \mu \mathrm{m}$ thick film of E7, sandwiched between two ITOcoated glass substrates, beforehand coated with a polyimide UV-photo-aligned for planar topologically-charged optical axis distribution. The ITO transparent conductive films work as electrodes for the application of an electric field to the liquid crystal. From now on, the former device will be referred to as $\mathrm{EOQP}_{1}$ and the latter as $\mathrm{EOQP}_{2}$. Adopting essentially the same apparatus as that described in Ref. 12, for both the EOQPs, we measured, as a function of the applied voltage, the powers of the converted $\left(P_{c}\right)$ and unconverted $\left(P_{u}\right)$ components of the output beam for an incident $\lambda=532 \mathrm{~nm}$ circularly polarized $\mathrm{TEM}_{00}$ laser beam. The temperature of both the EOQPs was maintained stable at $30^{\circ} \mathrm{C}$ during measurements. $P_{u}$ and $P_{c}$ are expected to depend on the optical retardation $\delta$ according to the Malus-like laws ${ }^{12}$ :

$$
P_{u}=P_{0} \cos ^{2}(\delta / 2), \quad P_{c}=P_{0} \sin ^{2}(\delta / 2),
$$

where $P_{0}$ is the total output power. In Fig. 1, the contrast ratios $\rho(v)=\left(P_{u}-P_{c}\right) /\left(P_{u}+P_{c}\right)=\cos \delta(v)$ are shown, for the rubbing-aligned and UV-photo-aligned EOQPs

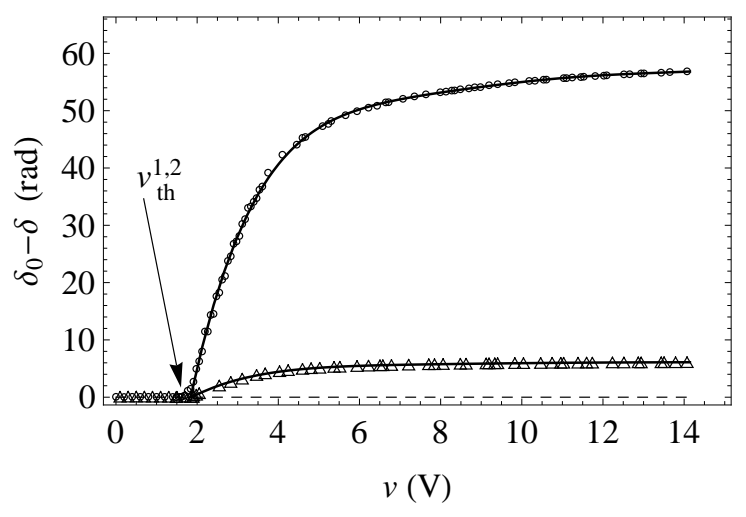

FIG. 2. The optical phase change $\delta(v)$ vs the applied voltage as extracted from the contrast $\rho(v)$ for $\operatorname{EOQP}_{1}(\circ)$ and $\mathrm{EOQP}_{2}(\triangle)$. The continuous lines correspond to sixth order polynomial curve obtained fitting the experimental data.

respectively, as functions of the rms values $v$ of the applied $1 \mathrm{kHz}$ AC voltage ${ }^{15}$. The dependence of $\delta(v)$ on the voltage arises from the torque exerted on the liquid crystal molecules by the applied electric field ${ }^{14}$. The behavior of $\delta(v)$ versus $v$, as deduced from $\rho(v)$, is shown in Fig. 2 for both devices. For $v<v_{t h}=1.85 \pm 0.01 \mathrm{~V}$, the nematic is undistorted in both the EOQPs and $\delta(v)=\delta_{0}=2 \pi \Delta n L / \lambda+\delta_{0}^{*}$, where $L$ stands for the thickness of the cell, $\Delta n=0.23$ is the intrinsic birefringence of the nematic E7 at $30^{\circ} \mathrm{C}$ and $\delta_{0}^{*}$ is a small constant residual birefringence. For $\mathrm{EOQP}_{1}, \delta_{0} \approx 1.7 \pi$ and for $\mathrm{EOQP}_{2} \delta_{0} \approx 0.8 \pi$. For $v=v_{t h}$ there is a discontinuity in the slope $\mathrm{d} \delta / \mathrm{d} v$. This is peculiar of a second order phase transition between the unperturbed and the distorted conformations at the critical voltage $v_{t h}$ (Freédericksz transition $)^{16}$. The theory of electro-optical effects in liquid crystals ${ }^{14,17}$ predicts $v_{t h}=\pi \sqrt{k_{11} / \epsilon_{0} \epsilon_{a}}$, where $k_{11}=9.2 \times 10^{-12} \mathrm{~N}\left(30^{\circ} \mathrm{C}\right)$ is the splay elastic constant of the liquid crystal ${ }^{18}, \epsilon_{0}$ is the vacuum dielectric constant and $\epsilon_{a}=14.3$ at $1 \mathrm{kHz}$ at $30^{\circ} \mathrm{C}{ }^{19}$. Actually, such expression returns $v_{t h} \lesssim 1 \mathrm{~V}$, i.e. half the experimental value. Such disagreement could be ascribed to the underlying simplified assumption of pure splay deformation. However, consistently with the theory, the experimental values of $v_{t h}$, within the experimental uncertainties, turn out to be the same for both EOQPs, which differ from one another in the thickness only. In the limit of high applied voltage, the overall change $\Delta=\delta_{0}-\delta(v)=\delta_{0}-\delta_{0}^{*}=2 \pi \Delta n L / \lambda$ is $\Delta \approx 56.8$ for $\mathrm{EOQP}_{1}$ and $\Delta \approx 6.1$ for $\mathrm{EOQP}_{2}$, yielding $L \approx 21 \mu \mathrm{m}$ for $\mathrm{EOQP}_{1}$ and $L \approx 2.3 \mu \mathrm{m}$ for $\mathrm{EOQP}_{2}$, respectively.

The different thickness of the cells is responsible for the different saturation values of $\delta(v)$ and for their different switching-off times. Assuming, for simplicity, that the initial director alignment is homogeneous, the switchingoff reorientation, in the small distortion limit, is expected to decay exponentially in time with a constant 


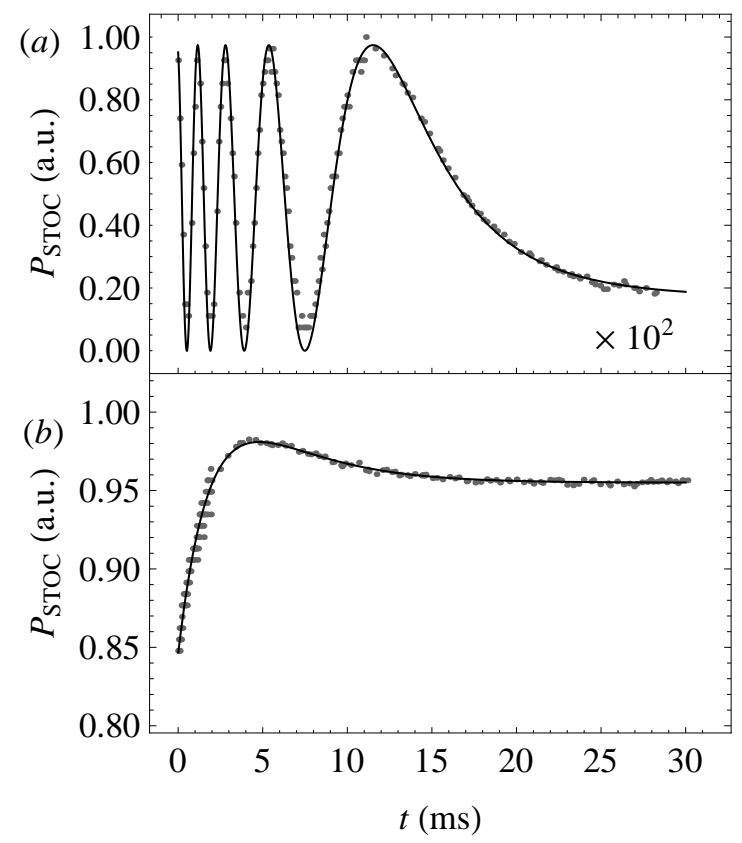

FIG. 3. Time decay of the power of the converted components of the output beam for $\operatorname{EOQP}_{1}(a)$ and $\operatorname{EOQP}_{2}(b)$ after the voltage switch-off. The starting voltages were $v_{1}=2.8 \mathrm{~V}$ for $\mathrm{EOQP}_{1}$ and $v_{2}=2.2 \mathrm{~V}$ for $\mathrm{EOQP}_{2}$. The continuous lines represent the theoretical behaviors. The time scale in panel $(a)$ is slower by a factor 100 with respect to panel $(b)$.

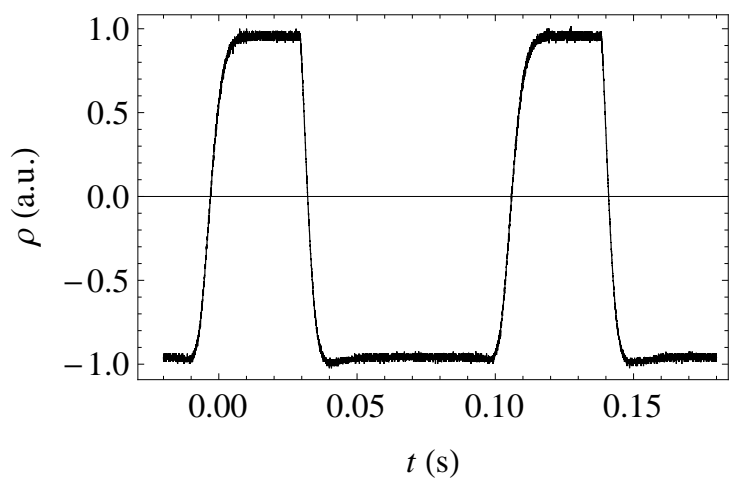

FIG. 4. Time behavior of contrast ratio $\rho$ for on-off switching of the applied voltage $\left(v_{o n}=3.2 \mathrm{~V}\right)$ for $\mathrm{EOQP}_{2}$.

$\tau=\frac{\gamma_{1}}{k_{11}}\left(\frac{L}{\pi}\right)^{2}$, where $\gamma_{1}=150 \mathrm{mPa}\left(30^{\circ} \mathrm{C}\right)$ is the rotational viscosity of the liquid crystal $^{20}$. The time behavior of the switching-off $P_{c}$ signals is consistent with such prediction and the measured decay time constants are $\tau_{1}=0.931 \pm 0.002 \mathrm{~s}$ and $\tau_{2}=(0.80 \pm 0.01) \times 10^{-2}$ s respectively (Fig. 3). These values are in reasonable agreement with those expected from theory, i.e. $\tau_{1}^{\text {theor }}=0.53 \mathrm{~s}$ and $\tau_{2}^{\text {theor }}=0.62 \times 10^{-2} \mathrm{~s}$. Nevertheless, consistently with the theoretical prediction for $\tau, \mathrm{EOQP}_{2}$, due to its reduced thickness, switches off much faster than $\mathrm{EOQP}_{1}$ and may be used for fast switching between different val-

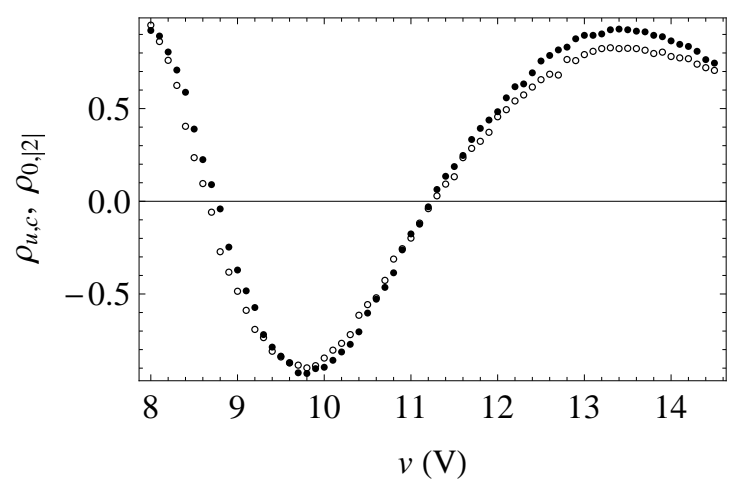

FIG. 5. Contrast ratio $\rho_{u, c}(v)$ vs voltage for unconvertedconverted components of the output sorted with respect to polarization $(\bullet)$. Contrast ratio $\rho_{0,|2|}(v)$ vs voltage for OAM $l=0$ and $l= \pm 2$ components of the output beam measured with Dove-Sagnac sorter (o). Data refer to EOQP 1 .

ues of $\delta$ as shown in Fig. 4. Finally, in order to check that the EOQPs actually work as SAM-to-OAM converters, the OAM of the output beam was directly measured adopting a method of sorting between $l=0$ and $l= \pm 2$ based on a polarizing Sagnac interferometer, with a Dove prism inserted along one of its arm, as elsewhere suggested $^{13}$. In Fig. 5, for EOQP 1 , the measured contrast ratio between the $l=|2|$ and $l=0$ components of the output beam was reported, for comparison, together with the contrast ratio between the converted and unconverted components respectively, as sorted with respect to their polarizations as above mentioned. The correlation between the two signal is $99.6 \%$.

In summary we realized an electro-optical SAMto-OAM converter, aimed not only at improving the handiness of classical QPs, but also at performing new tasks exploiting the newly introduced capability of EOQPs of managing superpositions of OAM eigenstates with $l=0, l=2$ and $l=-2$ in real-time for highdimensional qudits manipulation.

We acknowledge the financial support of the FETOpen programme within the $7^{\text {th }}$ Framework Programme of the European Commission, under grant number: 255914, Phorbitech.

${ }^{1}$ L. D. Landau and E. M. Lifsitz, Relativistic Quantum Theory, Pergamon Press, New York, 1960.

2 J. D. Jackson, Classical Electrodynamics, Wiley, New York, 1989.

${ }^{3}$ H. He, N. R. Heckenberg, and H. Rubinsztein-Dunlop, J. Mod. Opt. 42, 217 (1995).

${ }^{4}$ A. Mair, A. Vaziri, G. Welhs, and A. Zeilinger, Nature 412, 313 (2001).

${ }^{5}$ A. Muthukrishnan and C. R. S. Jr., Phys. Rev. A 62, 052309 (2000).

${ }^{6}$ B. P. Lanyon et al., Nat. Phys. 5, 134 (2008).

${ }^{7}$ I. Bregman, D. Aharonov, M. Ben-Or, and H. S. Eisenberg, Phys. Rev. A 77, 050301 (2008). 
${ }^{8}$ L. Marrucci, C. Manzo, and D. Paparo, Phys. Rev. Lett. 96, 163905 (2006).

${ }^{9}$ E. Nagali et al., Phys. Rev. Lett. 103, 013601 (2009).

${ }^{10}$ E. Nagali et al., Nat. Photon. 3, 720 (2009).

${ }^{11}$ E. Nagali, D. Giovannini, L. Marrucci, E. Santamato, and F. Sciarrino, Phys. Rev. Lett. 105, 073602;1 (2010).

${ }^{12}$ E. Karimi, B. Piccirillo, E. Nagali, L. Marrucci, and E. Santamato, Appl. Phys. Lett. 94, 231124 (2009).

${ }^{13}$ E. Karimi, S. Slussarenko, B. Piccirillo, L. Marrucci, and E. Santamato, Phys. Rev. A 81, 053813 (2010).

${ }^{14}$ P. G. de Gennes, The Physics of Liquid Crystals, Oxford University Press, Oxford, 1974.
${ }^{15} \mathrm{An} \mathrm{AC}$ rather than a DC voltage is applied to avoid electrochemical degradation ${ }^{17}$.

${ }^{16}$ V. Fréedericksz and V. Zolina, Trans. Faraday Soc. 29, 919 (1933).

${ }^{17}$ L. M. Blinov and V. G. Chigrinov, Electrooptic Effects in Liquid Crystal Materials, Springer, Berlin, 1996.

${ }^{18}$ H. Hakemi, Mol. Cryst. Liq. Cryst. 287, 215 (1996).

${ }^{19}$ H.-H. Liu and W. Lee, Appl. Phys. Lett. 97, 023510 (2010).

${ }^{20}$ Z. Ran, P. Zeng-Hui, L. Yong-Gang, Z. Zhi-Gang, and X. Li, Chin. Phys. B 18, 4380 (2009). 